\title{
ALTERNATING CATALAN NUMBERS AND CURVES WITH TRIPLE RAMIFICATION
}

\author{
GAVRIL FARKAS, RICCARDO MOSCHETTI, JUAN CARLOS NARANJO, AND GIAN PIETRO PIROLA
}

ABSTRACT. We determine the number of minimal degree covers of odd ramification for a general curve.

\section{INTRODUCTION}

The Catalan numbers $C_{n}:=\frac{1}{n+1}\left(\begin{array}{c}2 n \\ n\end{array}\right)$ form one of the most ubiquitous sequence in classical combinatorics. Stanley's book [St] lists 66 different manifestations of these numbers in various counting problems. In the theory of algebraic curves, the Catalan number $C_{n}$ counts the covers $C \rightarrow \mathbb{P}^{1}$ of minimal degree $n+1$ from a general curve $C$ of genus $2 n$. Each such cover has simple ramification and its monodromy group equals $S_{n+1}$. By degenerating $C$ to a rational $g$-nodal curve, it was already known to Castelnuovo $[\bar{C}]$ that the number of such covers coincides with the degree of the Grassmannian $G(2, n+2)$ in its Plücker embedding, which is well-known to equal $C_{n}$.

It has been shown by Guralnick and Magaard [GM] (see also [GS]) that for a general curve $C$ of genus $g>3$, the monodromy group $M_{f}$ of each cover $f: C \rightarrow \mathbb{P}^{1}$ is either the symmetric or the alternating group. For $g \leq 3$ several other groups do occur. The aim of this paper is to determine the number of covers $f: C \rightarrow \mathbb{P}^{1}$ with alternating monodromy having as source a general curve $C$ of genus $g$ and such that $\operatorname{deg}(f)$ is minimal (among covers with this property). The most natural case is when the local monodromy around each branch point is given by a 3-cycle. We refer to $f$ as being an odd cover. A moduli count indicates that $f$ has $3 g$ branch points and that $\operatorname{deg}(f)=2 g+1$. Writing $D=2\left(x_{1}+\cdots+x_{3 g}\right)$ for the ramification divisor of $f$, from the Hurwitz formula it follows that $\vartheta:=\mathscr{O}_{C}(D) \otimes f^{*} \mathscr{O}_{\mathbb{P} 1}(-1)$ is a theta characteristic on $C$. These coverings and their relation with spin structures have already been studied in [S1], [S2] and [F1]. We denote by $\mathscr{H}_{g}^{\text {odd }}$ the Hurwitz space parametrizing odd covers $f: C \rightarrow \mathbb{P}^{1}$ of degree $2 g+1$ with local monodromy at each branch point being given by a 3 -cycle. Fried showed in [F1] that $\mathscr{H}_{g}^{\text {odd }}$ has two connected components depending on the parity of $\vartheta$. The forgetful map

$$
\varphi: \mathscr{H}_{g}^{\text {odd }} \longrightarrow \mathscr{M}_{g}, \quad \varphi\left(\left[C \rightarrow \mathbb{P}^{1}\right]\right):=[C]
$$

is a map between varieties of the same dimension $3 g-3$. Using an inductive argument, it is shown in [MV] that $\varphi$ exists, hence $\varphi$ is generically finite. Our aim is to determine its degree $\mathfrak{A}_{g}:=\operatorname{deg}(\varphi)$. By analogy with the case of the symmetric group, we refer to $\mathfrak{A}_{g}$ as the $g$ th alternating Catalan number.

Theorem 1.1. The number of odd covers of degree $2 g+1$ of a general curve of genus $g \geq 3$ equals

$$
\mathfrak{A}_{g}=16^{g} \sum_{i=0}^{g}(-2)^{i}\left(\begin{array}{l}
g \\
i
\end{array}\right) C_{2 g-i} .
$$

Unlike the classical Catalan numbers, their alternating counterparts $\mathfrak{A}_{g}$ do not admit a closed formula. Instead, we determine their generating series.

Theorem 1.2. The generating series of the alternating Catalan numbers is the algebraic function

$$
\sum_{g \geq 0} \mathfrak{A}_{g} w^{2 g+1}=\frac{2 w}{\sqrt{1+64 w^{2}+16 w \sqrt{16 w^{2}+1}}+\sqrt{1+64 w^{2}-16 w \sqrt{16 w^{2}+1}}} .
$$


An elementary calculation shows that the convergence radius of the algebraic function appearing in Theorem[1.2 equals $\frac{\sqrt{2}}{16}$. Using [FS] Theorem IV.7, we can thus determine the exponential growth rate of the numbers $\mathfrak{A}_{g}$ and we have

$$
\mathfrak{A}_{g}=\left(\frac{16}{\sqrt{2}}\right)^{2 g+1} \chi(g), \quad \text { where } \quad \limsup _{g \rightarrow \infty} \sqrt[2 g+1]{\chi(g)}=1 .
$$

The proof of Theorem 1.2 relies on applying the Lagrange Inversion Theorem to the series of expressions computed in Theorem 1.1. Theorem[1.1 is proved by degenerating a general curve of genus $g$ to a flag curve $C$ consisting of a smooth rational spine having $g$ elliptic tails attached to general points of the spine. One can explicitly exhibit all odd admissible covers of degree $2 g+1$ having a source stably equivalent to $C$. This is carried out in Section 3 . The formula appearing in Theorem 1.1 depends on two initial values $N_{4}$ and $N_{5}$ which are related to the existence of certain odd maps of degrees 4 and 5 respectively on a general pointed elliptic curve $[E, P] \in \mathscr{M}_{1,1}$. Precisely $N_{5}$ counts the covers $E \stackrel{5: 1}{\rightarrow} \mathbb{P}^{1}$, which are totally ramified at $P$ and have three further triple ramification points. Similarly, $N_{4}$ is the number of degree 4 covers $E \stackrel{4: 1}{\rightarrow} \mathbb{P}^{1}$ having triple ramification at $P$ and at three further unassigned points. A significant part of the paper is devoted to proving that $N_{4}=N_{5}=16$, see Theorems 4.1 and 4.8. We present two independent proofs of this fact. The first, uses the theory of elliptic functions. Inspired by a method from [AP] we study the existence of such odd maps by counting the solutions of a certain differential equation on an elliptic curve. A Chern class calculation shows that $N_{4}, N_{5} \leq 16$. Then we show for a particular elliptic curve that the number of solutions is exactly 16 . The second proof of the equality $N_{4}=N_{5}=16$, is carried out in Section 5 , see Theorems 5.1 and 5.2 respectively. It relies on degeneration to a nodal elliptic curve and involves rather subtle intersection-theoretic calculations on moduli stacks of odd admissible covers.

Soon after the appearance of this paper, Lian's related work [Li] was posted on arXiv. He considers more general enumerative problems for pencils on curves than we do, though in the specific situation described in this paper his results are less explicit.

Acknowledgments: We are grateful to both M. Fried and D. Oprea for very useful discussions related to this circle of ideas.

Moschetti is member of GNSAGA (INDAM) and is partially supported by MIUR: Dipartimenti di Eccellenza Program (2018-2022)-Dept. of Math. Univ. of Pavia; Naranjo was partially supported by the Proyecto de Investigación MTM2015-65361-P; Pirola is member of GNSAGA (INDAM) and is partially supported by PRIN Project Moduli spaces and Lie theory (2017) and by MIUR: Dipartimenti di Eccellenza Program (2018-2022) Dept. of Math. Univ. of Pavia. Farkas was supported by the DFG Grant Syzygien und Moduli.

\section{Preliminaries}

We collect a few things that will be used throughout the paper.

2.1. Monodromy of coverings and Hurwitz spaces of odd covers. Let $f: C \rightarrow \mathbb{P}^{1}$ be a finite cover of degree $d$ and denote by $B:=\left\{P_{1}, \ldots, P_{n}\right\}$ its branch locus. For a point $Q \in \mathbb{P}^{1} \backslash B$, let

$$
\rho_{f}: \pi_{1}\left(\mathbb{P}^{1} \backslash B, Q\right) \rightarrow S_{d}
$$

be its monodromy representation. We denote by $M_{f}:=\operatorname{Im}\left(\rho_{f}\right)$ the monodromy group of $f$. The local monodromy of $f$ around a branch point $P_{i} \in B$ is given by $\tau_{i}:=\rho_{f}\left(\left[\gamma_{i}\right]\right) \in S_{d}$, where $\gamma_{i}$ is a simple loop around $P_{i}$ based at $Q$. The cover $f$ is said to be alternating if $M_{f} \subseteq A_{d}$. We shall often consider alternating covers $f: C \rightarrow \mathbb{P}^{1}$, such that each local monodromy $\tau_{i}$ is given by an odd cycle. We refer to such an $f$ as being an odd cover.

We denote by $\mathscr{H}_{g}^{\text {odd }}$ the Hurwitz space parametrizing odd covers $f: C \rightarrow \mathbb{P}^{1}$ of degree $2 g+1$ branched at $3 g$ points. We require that the local monodromy around each branch point of $f$ be given by a 3 -cycle. Such a cover is endowed with a theta characteristics $\vartheta:=\mathscr{O}_{C}(D) \otimes f^{*}\left(\mathscr{O}_{\mathbb{P}}(-1)\right)$, 
where $D$ is the half of the ramification divisor $R_{f}$. As proved by Mumford (see $[\mathrm{Mu}]$ ), the parity of the spin structure $\vartheta$ is a deformation invariant. Two odd covers $f_{1}: C_{1} \rightarrow \mathbb{P}^{1}$ and $f_{2}: C_{2} \rightarrow \mathbb{P}^{1}$ are identified as points in $\mathscr{H}_{g}^{\text {ord }}$ when there exists an isomorphism $\tau: C_{1} \rightarrow C_{2}$ and an automorphism $\bar{\tau}: \mathbb{P}^{1} \rightarrow \mathbb{P}^{1}$ such that $f_{2} \circ \tau=\bar{\tau} \circ f_{1}$. We denote by $\left[f: C \rightarrow \mathbb{P}^{1}\right] \in \mathscr{H}_{g}^{\text {ord }}$ the moduli point of the cover $f$.

Let $\overline{\mathscr{H}}_{g}^{\text {odd }}$ be the compactification of $\mathscr{H}_{g}^{\text {odd }}$ by admissible $A_{2 g+1}$-covers. By [ACV], the stack $\overline{\mathscr{H}}_{g}^{\text {odd }}$ is isomorphic to the stack of balanced twisted stable maps into the classifying stack $\mathscr{B} A_{2 g+1}$, that is,

$$
\overline{\mathscr{H}}_{g}^{\text {odd }}:=\overline{\mathscr{M}}_{0,3 g}\left(\mathscr{B} A_{2 g+1}\right) / S_{3 g} \text {, }
$$

where the action of the symmetric group $S_{3 g}$ is given by permuting the branch points. For details concerning the construction of the space of admissible covers we refer to [ACV]. Note that $\overline{\mathscr{H}}_{g}^{\text {odd }}$ is the normalization of the space $\mathscr{H} \mathscr{M}_{\mathrm{g}}^{\text {odd }}$ of Harris-Mumford admissible covers introduced in [HM].

Points of $\overline{\mathscr{H}}_{g}^{\text {odd }}$ are odd admissible coverings $\left[f: X \rightarrow \Gamma, P_{1}+\cdots+P_{3 g}\right]$, where $X$ and $\Gamma$ are nodal curves of genus $g$ and 0 respectively, $f$ is a finite map of degree $2 g+1$ with $f^{-1}\left(\Gamma_{\text {sing }}\right)=X_{\text {sing }}$ and $P_{1}, \ldots, P_{3 g} \in \Gamma_{\text {reg }}$ are the branch points of $f$. The local monodromy of $f$ around $P_{i} \in \Gamma$ is given by a 3-cycle $\tau_{i} \in A_{2 g+1}$, for $i=1, \ldots, 3 g$. The local monodromy of $f$ at both branches of $X$ at a node $p \in X_{\text {sing }}$ is given by an alternate permutation, which is not necessarily a 3 -cycle. We denote by

$$
\varphi: \overline{\mathscr{H}}_{g}^{\text {odd }} \rightarrow \overline{\mathscr{M}}_{g}
$$

the map associating to an admissible cover $\left[f: X \rightarrow \Gamma, P_{1}+\cdots+P_{3 g}\right]$ the stable model st $(X)$ of its source. As discussed in the Introduction, $\varphi$ is a generically finite map.

We discuss the local structure of the space of admissible covers following [HM $]$ p.62. We fix a point $\xi:=\left[f: X \rightarrow \Gamma, P_{1}+\cdots+P_{3 g}\right]$ as above and assume $\Gamma_{\text {sing }}=\left\{u_{1}, \ldots, u_{r}\right\}$. For $i=1, \ldots, r$, set $f^{-1}\left(u_{i}\right)=\left\{Q_{i, 1}, \ldots, Q_{i, \ell_{i}}\right\} \subseteq X_{\text {sing. }}$. The (non-normalized) space $\mathscr{H} \mathscr{M}_{\mathrm{g}}^{\text {odd }}$ is described by its local ring

$$
\hat{O}_{\xi, \mathscr{H}^{\prime} \mathscr{M}_{g}^{\text {odd }}}=\mathbb{C}\left[\left[t_{1}, \ldots, t_{3 g-3}, s_{i, 1}, \ldots, s_{i, \ell_{i}}, \quad i=1, \ldots, r\right]\right] / s_{i, 1}^{\mu_{i, 1}}=\cdots=s_{i, \ell_{i}}^{\mu_{i, \ell_{i}}}=t_{i}, \quad i=1, \ldots, r,
$$

where $t_{i}$ is the local corresponding to smoothing the node $u_{i} \in \Gamma$ and $\left(\mu_{i, 1}, \ldots, \mu_{i, \ell_{i}}\right)$ describes the ramification profile of $f^{-1}\left(u_{i}\right)$. In particular, $\mathscr{H} \mathscr{M}_{g}^{\text {odd }}$ (and hence $\overline{\mathscr{H}}_{g}^{\text {odd }}$ ) is smooth at $t$ whenever over each node $u_{i}$ with $i=1, \ldots, r$ there exist at most one ramification point, that is, at most one index $j \in\left\{1, \ldots, \ell_{i}\right\}$ with $\mu_{i, j}>1$.

2.2. Schubert cycles with respect to osculating flags to rational normal curves. We recall the definition of Schubert cycles in the Grassmannian of lines $\mathbb{G}:=G(2, V)$, where $V \cong \mathbb{C}^{n}$. After choosing a flag $F_{\bullet}: V=V_{n} \supset V_{n-1} \supset \ldots \supset V_{0}=0$, for a decreasing sequence of positive integers $\mu:=\left(\alpha_{1} \geq \alpha_{0}\right)$ we introduce the Schubert cycle

$$
\sigma_{\mu}=\sigma_{\mu}\left(F_{\bullet}\right):=\left\{\Lambda \in \mathbb{G}: \Lambda \subseteq V_{n-\alpha_{0}}, \Lambda \cap V_{n-\alpha_{1}-1} \neq 0\right\} .
$$

When the meaning of the flag $F_{0}$ is clear from the context, we shall drop it from the notation of the corresponding Schubert cycle. Note that $\operatorname{codim}\left(\sigma_{\mu}, \mathbb{G}\right)=|\mu|=\alpha_{0}+\alpha_{1}$.

When counting admissible covers we often use non-generic flags defined in terms of a rational normal curve $R \subseteq \mathbb{P}^{n-1}$ embedded by $V:=H^{0}\left(\mathbb{P}^{1}, \mathscr{O}_{\mathbb{P}^{1}}(n-1)\right)$. For a point $P \in R$, let $F_{\mathbf{0}}(P)$ be the osculating flag of $R$ at $P$, thus $V_{i}:=H^{0}\left(\mathbb{P}^{1}, \mathscr{O}_{\mathbb{P} 1}(n-1)(-(n-i) P)\right)$ for $i=0, \ldots, n-1$. The osculating flags to $R$ enjoy two very desirable transversality properties:

(i) For any number of distinct points $P_{1}, \ldots, P_{s} \in R$ and any partitions $\mu_{1}, \ldots, \mu_{s}$, the intersection $\cap_{i=1}^{s} \sigma_{\mu_{i}}\left(F_{\bullet}\left(P_{i}\right)\right)$ has the expected dimension 2(n-2)-|$\mu_{1}|-\cdots-| \mu_{s} \mid$, see [EH1], Theorem 2.3. 
(ii) If all the points $P_{i}$ are in $\mathbb{P}^{1}(\mathbb{R})$ and $\left|\mu_{1}\right|+\cdots+\left|\mu_{s}\right|=2(n-2)$, then the intersection $\cap_{i=1}^{s} \sigma_{\mu_{i}}\left(F_{\bullet}\left(P_{i}\right)\right)$ is a reduced union of real points, see [MTV].

2.3. Odd covers and differential equations on elliptic curves. Let $E$ be a complex elliptic curve and fix a point $P \in E$. We consider the group structure on $E$ having the point $P \in E$ as origin. We have $E \cong \mathbb{C} / \Lambda$, where $\Lambda$ is a lattice generated by 1 and $\tau$, where $\operatorname{Im}(\tau)>0$. Let $\pi: \mathbb{C} \rightarrow E$ be the universal covering, so that $\pi(0)=P$ and we denote by $\sigma: E \rightarrow E$ the involution fixing $P$, which can be thought as the involution associated to the hyperelliptic linear series $|2 P|$, which induces a map $h: E \rightarrow \mathbb{P}^{1}$. We write $D:=P+Q+R+S$ for the ramification divisor of $h$, thus $Q, R$ and $S$ are the point of order two on $E$. The function $h$ is determined explicitly by the Weierstrass function $\wp$ (see [AMS] and [La]), which is given by

$$
\wp(z)=\frac{1}{z^{2}}+\frac{1}{20} g_{2} z^{2}+\frac{1}{28} g_{3} z^{4}+O\left(z^{6}\right),
$$

where $g_{2}$ and $g_{3}$ depend on the choice of the lattice $\Lambda$. Consider the image of the half period

$$
e_{1}:=\wp\left(\frac{1}{2}\right), e_{2}:=\wp\left(\frac{\tau}{2}\right), e_{3}:=\wp\left(1+\frac{\tau}{2}\right) .
$$

We record the following relations between $\wp$ and its derivatives

$$
\begin{aligned}
& \wp^{\prime}(z)^{2}=4 \wp(z)^{3}-g_{2} \wp(z)-g_{3}=4\left(\wp(z)-e_{1}\right)\left(\wp(z)-e_{2}\right)\left(\wp(z)-e_{3}\right), \\
& \wp^{\prime \prime}(z)=6 \wp(z)^{2}-\frac{1}{2} g_{2} .
\end{aligned}
$$

The Weierstrass form of $E$ is given by $y^{2}=4 x^{3}-g_{2} x-g_{3}=4\left(x-e_{1}\right)\left(x-e_{2}\right)\left(x-e_{3}\right)$ and the $j$ invariant of $E$ is computed by the well-known formula

$$
j_{E}=1728 \frac{g_{2}^{3}}{g_{2}^{3}-27 g_{3}^{2}} \text {. }
$$

The field of the rational function $\mathbb{C}(E)$ is isomorphic to the subfield $\mathbb{C}\left(\wp, \wp^{\prime}\right)$ of the complex meromorphic functions generated by $\wp$ and $\wp^{\prime}$, see [La].

As described in the Introduction, an odd map $f: E \rightarrow \mathbb{P}^{1}$ comes equipped with a spin structure $\vartheta=\mathscr{O}_{E}(D) \otimes f^{*} \mathscr{O}_{\mathbb{P}}(-1) \in \operatorname{Pic}^{0}(E)$, where $D:=\frac{1}{2} R_{f}$ is half of the ramification divisor $R_{f}$ of $f$. Hence there are four possibilities, meaning

$$
\vartheta=\mathscr{O}_{E}, \vartheta=\mathscr{O}_{E}(P-Q), \vartheta=\mathscr{O}_{E}(P-R), \vartheta=\mathscr{O}_{E}(P-S) .
$$

We fix a non-trivial holomorphic form $d z$ in the space of holomorphic differentials $H^{0}\left(E, \omega_{E}\right)$. We may assume that $h$, viewed as a meromorphic function, has a second order pole at $P$ and a second order zero at $Q$. The next proposition allows us to translate the computation of the quantities $N_{4}$ and $N_{5}$, essential in proving Theorem 1.1 into finding the solutions of certain differential equations on $E$.

Proposition 2.1. Let $\vartheta \in \operatorname{Pic}^{0}(E)[2]$. A meromorphic function $f$ corresponds to an odd cover $f: E \rightarrow$ $\mathbb{P}^{1}$ with associated spin structure $\vartheta$ if and only if there exists a meromorphic function $s$ on $E$ with

$$
d f=s^{2} \omega,
$$

where $\omega=d z$ if $\vartheta \cong \mathscr{O}_{E}$, and $\omega=h d z$ if $\vartheta \cong \mathscr{O}_{E}(P-Q)$ respectively.

Proof. Assume $f: E \rightarrow \mathbb{P}^{1}$ is an odd function with trivial spin structure $\vartheta \cong \mathscr{O}_{E}$. Let $A:=f^{*}(\infty)$ be the divisor of poles of $f$. Since $D-A$ is a principal divisor, there exists a meromorphic function $s \in \mathbb{C}(E)$ with

$$
\operatorname{div}(s)=D-A \text {. }
$$


Then, $\operatorname{div}\left(s^{2}\right)=2 D-2 A=R_{f}-2 A$, which is precisely the divisor of $d f$. Up to modifying $s$ by a constant, the equation $d f=s^{2} d z$ is satisfied. In the opposite direction, if $f$ satisfies equation (4), then by taking local coordinates it is clear that $f$ is odd.

The case when the associated spin structure $\vartheta$ is even is similar. With the same notation we have that $D-A$ is linearly equivalent to $Q-P$, hence there exists $s \in \mathbb{C}(E)$ with

$$
\operatorname{div}(s)=D-A+P-Q .
$$

Therefore, $\operatorname{div}\left(s^{2} h\right)=R_{f}-2 A$, since $\operatorname{div}(h)=2 Q-2 P$. As above, after rescaling we may assume that $d f=s^{2} h d z$. Assuming conversely that $f$ satisfies this equation, a simple local analysis shows that $f$ is an odd function.

Remark 2.2. Proposition2.1 is valid for odd covers of arbitrary genus. Consider a curve $C$ of genus $g$ and a theta characteristic $\vartheta$ on $C$. Then a cover $f: C \rightarrow \mathbb{P}^{1}$ of degree $2 g+1$ is odd with associated spin structure $\vartheta$ if and only if there exists a divisor $A$ of degree $2 g+1$ such that $d f=s^{2} \in H^{0}\left(C, \omega_{C}(2 A)\right)$, for $s \in H^{0}(C, \vartheta(A))$.

In Section 4 we shall study the solutions of the equation (4) when $\operatorname{deg}(f)=4$ and $f$ has a triple ramification point at $P$ and when $\operatorname{deg}(f)=5$ and $P$ is a point of total ramification of $f$ respectively.

\section{ODD ADMISSIBLE COVERS ON FLAG CURVES OF GENUS $g$}

In this section we apply degeneration methods in order to prove the formula (3.2) below. This is an intermediate step in the proof of the main Theorem (1.1). We recall that for a pencil $\ell \in G_{d}^{1}(C)$ on a smooth curve $C$, for a point $P \in C$ we denote by $a^{\ell}(P)=\left(a_{0}^{\ell}(P)<a_{1}^{\ell}(P)\right)$ its vanishing sequence at $P$ and by $\alpha^{\ell}(P)=\left(\alpha_{0}^{\ell}(P)=a_{0}^{\ell}(P), \alpha_{1}^{\ell}(P)=a_{1}^{\ell}(P)-1\right)$ its ramification sequence. We fix a general pointed elliptic curve $[E, P] \in \mathscr{M}_{1,1}$ and we introduce two loci. Firstly,

$G_{4, \mathrm{tr}}^{1}(E, P):=\left\{\ell \in G_{4}^{1}(E): a_{1}^{\ell}(P) \geq 3\right.$, there exist distinct points $P_{i} \in E \backslash\{P\}$ with $\left.a_{1}^{\ell}\left(P_{i}\right) \geq 3, i=1,2,3\right\}$. A pencil $\ell \in G_{4, \mathrm{tr}}^{1}(E, p)$ corresponds to a cover $f: E \rightarrow \mathbb{P}^{1}$ of degree 4 ramified triply at $P, P_{1}, P_{2}, P_{3}$ and having no further ramification points. Secondly, we define the locus

$G_{5, \mathrm{tr}}^{1}(E, P):=\left\{\ell \in G_{5}^{1}(E): a_{1}^{\ell}(P) \geq 5\right.$, there exist distinct points $P_{i} \in E \backslash\{P\}$ with $\left.a_{1}^{\ell}\left(P_{i}\right) \geq 3, i=1,2,3\right\}$. A pencil $\ell \in G_{5, \text { tr }}^{1}(E, P)$ corresponds to a cover $f: E \rightarrow \mathbb{P}^{1}$ totally ramified at $P$, triply ramified at $P_{i}$ for $i=1,2,3$ and having no further ramification points.

A parameter count yields that both $G_{4, \mathrm{tr}}^{1}(E, P)$ and $G_{5, \mathrm{tr}}^{1}(E, P)$ are 0 -dimensional and we denote

$$
N_{4}:=\left|G_{4, \mathrm{tr}}^{1}(E, P)\right| \text { and } N_{5}:=\left|G_{5, \mathrm{tr}}^{1}(E, P)\right|
$$

respectively, their cardinalities. We shall later prove that both $G_{4, \mathrm{tr}}^{1}(E, P)$ and $G_{5, \mathrm{tr}}^{1}(E, P)$ are reduced, but for now we do not need that.

We fix once and for all a flag curve

$$
\left[C:=R \cup_{Q_{1}} E_{1} \cup \ldots \cup_{Q_{g}} E_{g}\right] \in \overline{\mathscr{M}}_{g}
$$

consisting of a smooth rational curve $R$ and $g$ elliptic tails $E_{i}$ meeting the spine $R$ at the point $Q_{j}$ for $j=1, \ldots, g$. We require that $\left[E_{j}, Q_{j}\right] \in \mathscr{M}_{1,1}$ are general, which in practice means that $E_{j}$ is not isomorphic to the Fermat cubic. The use of such flag curves in proving the classical Brill-Noether Theorem is well documented, see [EH2].

Theorem 3.1. The fibre of the morphism $\varphi: \overline{\mathscr{H}}_{g}^{\text {odd }} \rightarrow \overline{\mathscr{M}}_{g}$ over the point $[C]$ can be described as

$$
\varphi^{-1}([C])=\bigcup_{J \subseteq\{1, \ldots, g\}}\left(\prod_{j \in J} G_{4, \mathrm{tr}}^{1}\left(E_{j}, Q_{j}\right) \times \prod_{i \in J^{c}} G_{5, \mathrm{tr}}^{1}\left(E_{i}, Q_{i}\right)\right) \times\left(\bigcap_{j \in J} \sigma_{3,1}\left(F_{\bullet}\left(Q_{j}\right)\right) \cap \bigcap_{i \in J^{c}} \sigma_{4,0}\left(F_{\bullet}\left(Q_{i}\right)\right)\right) .
$$


Futhermore, if the points $Q_{i} \in R$ are chosen generically, the above cycle is 0 -dimensional and reduced.

An immediate consequence of Theorem 3.1 is the following formula for the alternating Catalan number $\mathfrak{A}_{g}:=\operatorname{deg}(\varphi)$.

Theorem 3.2. The number of odd coverings of degree $2 g+1$ in a generic curve of genus $g \geq 3$ is

$$
\mathfrak{A}_{g}=\left(N_{4} \sigma_{4,0}+N_{5} \sigma_{3,1}\right)^{g} \in H^{\mathrm{top}}(G(2,2 g+2), \mathbb{Z}) .
$$

Proof. In the statement of Theorem 3.1, we sum over $k:=|J| \leq g$ to obtain

$$
\mathfrak{A}_{g}=\sum_{k=0}^{g}\left(\begin{array}{l}
g \\
k
\end{array}\right) N_{4}^{k} N_{5}^{g-k} \sigma_{3,1}^{k} \cdot \sigma_{4,0}^{g-k}=\left(N_{4} \sigma_{4,0}+N_{5} \sigma_{3,1}\right)^{g} \in H^{\text {top }}(G(2,2 g+2), \mathbb{Z}) .
$$

Proof of Theorem 3.1 . We start with an odd admissible cover $\left[f: X \rightarrow \Gamma, P_{1}+\cdots+P_{3 g}\right]$ of degree $2 g+1$, having as source a nodal curve $X$ stably equivalent to $C$. For $i=1, \ldots, 3 g$, we denote by $x_{i} \in f^{-1}\left(P_{i}\right)$ the unique odd ramification point lying over the branch point $P_{i}$. We fix an index $j \in\{1, \ldots, g\}$ and consider the restriction $f_{j}=f_{\mid E_{j}}: E_{j} \rightarrow \mathbb{P}^{1}$, where $\mathbb{P}^{1}$ is one of the components of $\Gamma$. Let $a=a_{1}^{f_{j}}\left(Q_{j}\right)$ be the vanishing index of the point of attachment $Q_{j}$ and note that away from $Q_{j}$, the ramification points of $f_{j}$ are precisely those points $x_{i}$ with $i=1, \ldots, 3 g$ which lie on $E_{j}$. Set $d_{j}:=\operatorname{deg}\left(f_{j}\right)$.

We claim that at least three of the odd ramification points of $f$ lie on $E_{j}$. Indeed, assume first that, on the contrary, at most one such point lies on $E_{j}$. By the Hurwitz formula, then $2 d_{j}=\operatorname{deg}\left(R_{f_{j}}\right)=$ $a-1+2=a+1 \leq d_{j}+1$, hence $d_{j}=1$, which is impossible. If two odd ramification points lie on $E_{j} \backslash\left\{Q_{j}\right\}$, then by the same reasoning $2 d_{j}=a-1+2+2 \leq d_{j}+3$, hence $d_{j}=3$. But then $f_{j}: E_{j} \rightarrow \mathbb{P}^{1}$ is a degree 3 cover having three total ramification points, which forces $E_{j}$ to be isomorphic to the Fermat cubic, in particular to have $j$-invariant zero, contradicting the generality of $E_{j}$. Thus at least three of the points $x_{1}, \ldots, x_{3 g}$ specialize on each tail $E_{j}$. Since there are precisely $g$ elliptic tails, this implies that precisely three ramification points lie on each of $E_{1}, \ldots, E_{g}$, whereas the spine $R$ contains no ramification points.

Applying once more the Hurwitz formula to $f_{j}$, we get $2 d_{j}-2=a-1+6 \leq d_{j}+5$. If $d_{j}=5$, then $a=5$ and the pencil corresponding to $f_{j}$ belongs to $G_{5, \text { tr }}^{1}\left(E_{j}, Q_{j}\right)$, in particular there are $N_{5}$ choices for $f_{j}$. If, on the other hand, $d_{j}=4$, then $a=3$ and the pencil corresponding to $f_{j}$ gives rise to a point in $G_{4, \mathrm{rr}}^{1}\left(E_{j}, Q_{j}\right)$. Let $J \subseteq\{1, \ldots, g\}$ be the set of labels for the elliptic curves $E_{j}$ with $d_{j}=4$, in which case $J^{c}$ contains the labels for those elliptic tails $E_{j}$ having $d_{j}=5$. Set $|J|=: k \leq g$. For each $j \in J$, writing $f_{j}^{-1}\left(f_{j}\left(Q_{j}\right)\right)=\left\{Q_{j}, Q_{j}^{\prime}\right\}$, it follows that there exists a rational component $R_{j}^{\prime}$ of $X$ meeting $E_{j}$ at $Q_{j}^{\prime}$ and such that $f\left(R_{j}^{\prime}\right)=f(R)$. In fact $\operatorname{deg}\left(f_{\mid R_{j}^{\prime}}\right)=1$. It follows that the degree of the restriction $f_{R}=f_{\mid R}: R \rightarrow \mathbb{P}^{1}$ is then at most $2 g+1-|J|=2 g+1-k$. Since the ramification indices at $Q_{j}$ on the two branches of $R$ and $E_{j}$ must agree for $j=1, \ldots, g$, it follows that $f_{R}$ corresponds to a pencil $\ell_{R} \in G_{d}^{1}(R)$ having vanishing $a_{1}^{\ell_{R}}\left(Q_{i}\right) \geq 5$, for $i \in J^{c}$ and $a_{1}^{\ell_{R}}\left(Q_{j}\right) \geq 3$ for $j \in J$. The Hurwitz formula applied to $f_{R}$ implies that $d \geq 2 g+1-k$, hence $d=2 g+1-k$. Equivalently, the pencil

$$
\ell:=\ell_{R}\left(\sum_{j \in J} Q_{j}\right) \in G_{2 g+1}^{1}(R)
$$

obtained from $\ell_{R}$ by adding base points at all points with labels from $J$ satisfies $a^{\ell}\left(Q_{j}\right) \geq(1,4)$ for $j \in J$ and $a^{\ell}\left(Q_{i}\right) \geq(0,5)$ for all $i \in J^{c}$.

Write $\ell=\left(\mathscr{O}_{\mathbb{P}}(2 g+1), V\right)$, for a subspace of sections $V \in G\left(2, H^{0}\left(\mathbb{P}^{1}, \mathscr{O}_{\mathbb{P}^{1}}(2 g+1)\right)\right)=G(2,2 g+2)$. We regard $R$ as a rational normal curve in $\mathbb{P}^{2 g+1}$ embedded each point by $H^{0}\left(R, \mathscr{O}_{R}(2 g+1)\right)$ and denote by $F_{0}\left(Q_{j}\right)$ the osculating flag at $Q_{j}$. Then for each vanshing sequence $\left(a_{0}<a_{1}\right)$ the condition $a^{\ell}\left(Q_{j}\right) \geq$ $\left(a_{0}, a_{1}\right)$ is equivalent to $V \in \sigma_{a_{1}-1, a_{0}}\left(F_{\bullet}\left(Q_{j}\right)\right)$, which establishes the Theorem set-theoretically. 
Observe that all covers $[f] \in \varphi^{-1}([C])$ correspond to smooth points of $\overline{\mathscr{H}}_{g}^{\text {odd }}$. Indeed the rational target curve $\Gamma=f(X)$ has $g+1$ components, namely $f(R)$ and $f\left(E_{j}\right)$ where $j=1, \ldots, g$. Over each node $f\left(Q_{j}\right) \in \Gamma_{\text {sing }}$ lies a single ramification point, which using the local description (1) implies that $\overline{\mathscr{H}}_{g}^{\text {odd }}$ is smooth at $[f]$. Furthermore, the fibre $\varphi^{-1}([C])$ is scheme-theoretically isomorphic to disjoint unions of copies of the intersection of Schubert cycles

$$
\left(\bigcap_{j \in J} \sigma_{3,1}\left(F_{\bullet}\left(Q_{j}\right)\right) \cap \bigcap_{i \in J^{c}} \sigma_{4,0}\left(F_{\bullet}\left(Q_{i}\right)\right)\right) .
$$

Following [MTV] this intersection is transverse when the points $Q_{j} \in R$ are general, which finishes the proof.

\section{COUNTING ODD COVERS OF ELLIPTIC CURVES I: AN APPROACH VIA DIFFERENTIAL EQUATIONS}

The goal of this Section is to determine the quantities $N_{4}$ and $N_{5}$ appearing in Theorem 3.2. Thanks to Proposition 2.1 these two problems can be reformulated in terms of differential equations of type (4). The result will follow by combining the upper bound provided by Proposition 4.5 and the lower bound provided by Proposition 4.7 .

4.1. Odd covers of degree 4 on an elliptic curve. We will ultimately prove the following result:

Theorem 4.1. The number $N_{4}$ of odd maps $f: E \rightarrow \mathbb{P}^{1}$ of degree 4 from a general pointed elliptic curve $[E, P] \in \mathscr{M}_{1,1}$ is equal to 16 .

We shall use the same terminology of Subsection 2.3. The origin $P$ of the elliptic curve $E$ may be assumed to be one of the ramification points, hence the odd function $f$ we are looking for belongs to $H^{0}\left(E, \mathscr{O}_{E}(3 P+x)\right)$, for some point $x \in E$. Assume first $x \neq P$. By considering the local expression of $f$ around $P$ and $x$ and taking derivatives, we obtain that $d f$ has a pole of order 4 at $P$ and a pole of order 2 at $x$, that is, $d f \in H^{0}\left(E, \mathscr{O}_{E}(4 P+2 x)\right)$. Differentiation provides a linear map

$$
\delta_{x}: H^{0}\left(E, \mathscr{O}_{E}(3 P+x)\right) \rightarrow H^{0}\left(E, \omega_{E}(4 P+2 x)\right) \cong H^{0}\left(E, \mathscr{O}_{E}(4 P+2 x)\right) .
$$

This analysis also works when $x=P$. Then $H^{0}\left(E, \mathscr{O}_{E}(4 P+2 x)\right)=H^{0}\left(E, \mathscr{O}_{E}(6 P)\right)$; the function $f$ has a pole of order 4 at $P$ and so $d f$ has there a pole of order 5 at $P$ and $d f \in H^{0}\left(E, \mathscr{O}_{E}(5 P)\right)$, which lies inside $H^{0}\left(E, \mathscr{O}_{E}(6 P)\right)$.

Consider now a meromorphic function $s$ satisfying equation (4). If the associated theta characteristic $\vartheta$ is trivial, we have $s \in H^{0}\left(E, \mathscr{O}_{E}(2 P+x)\right.$ ), and we can consider the (non-linear) map

$$
\begin{aligned}
\alpha_{x}: H^{0}\left(E, \mathscr{O}_{E}(2 P+x)\right) & \rightarrow H^{0}\left(E, \mathscr{O}_{E}(4 P+2 x)\right) \\
s & \mapsto s^{2} d z .
\end{aligned}
$$

If $\vartheta \cong \mathscr{O}_{E}(P-Q)$, we consider a similar map $\alpha_{x}: H^{0}\left(E, \mathscr{O}_{E}(P+Q+x)\right) \rightarrow H^{0}\left(E, \mathscr{O}_{E}(4 P+2 x)\right)$ defined by $\alpha_{x}(s):=s^{2} h d z$. The following corollary relates the maps $\delta_{x}$ and $\alpha_{x}$.

Corollary 4.2. The solutions of equation (4) coincides with the intersection of the images of the maps $\delta_{x}$ and $\alpha_{x}$, as $x \in E$ varies.

Proof. This follows directly from the proof of Proposition 2.1. The map $\delta_{x}$ correspond to the left hand side of Equation (4), while the map $\alpha_{x}$ correspond to the right hand side.

In order to exploit this result, notice that the map $\delta_{x}$ is linear, so in order to intersect its image with the one of $\alpha_{x}$ it is convenient to look at the kernel of the following composition

$$
H^{0}\left(E, \mathscr{O}_{E}(2 P+x)\right) \stackrel{\alpha_{x}}{\longrightarrow} H^{0}\left(E, \mathscr{O}_{E}(4 P+2 x)\right) \rightarrow \frac{H^{0}\left(E, \mathscr{O}_{E}(4 P+2 x)\right)}{\delta_{x}\left(H^{0}\left(E, \mathscr{O}_{E}(3 P+x)\right)\right.} .
$$


We regard these maps globally by moving the point $x \in E$. To that end, we consider the projections $\pi_{i}: E \times E \rightarrow E$ for $i=1,2$ and the diagonal $\Delta \subseteq E \times E$. For a point $x \in E$, define $E_{x}$ as $E \times\{x\}:=\pi_{2}^{*}(x)$. For an effective divisor $A=\sum n_{i} P_{i}$ on $E$, we set $E_{A}:=\sum n_{i} E_{P_{i}}$.

Definition 4.3. For an integer $m$ and an effective divisor $A$ on $E$, we define the vector bundle $D_{m, A}:=$ $\pi_{1 *}\left(\mathscr{O}_{E \times E}\left(m \Delta+E_{A}\right)\right)$ on $E$.

Observe that $\mathscr{G}:=D_{1,3 P}, \mathscr{F}:=D_{1,2 P}$ and $\mathscr{U}:=D_{2,4 P}$ are the vector bundles we mentioned before. We have the natural identifications for the respective fibres

$$
\mathscr{G}(x) \cong H^{0}\left(E, \mathscr{O}_{E}(3 P+x)\right), \mathscr{F}(x) \cong H^{0}\left(E, \mathscr{O}_{E}(2 P+x)\right) \text { and } \mathscr{U}(x) \cong H^{0}\left(E, \mathscr{O}_{E}(4 P+2 x)\right) .
$$

The map $\delta_{x}$ correspond to a sheaf morphism $\delta: \mathscr{G} \rightarrow \mathscr{U}:$ start by considering the differential

$$
d: \mathscr{O}_{E \times E} \rightarrow \Omega_{E \times E}^{1}=\pi_{1}^{*} \mathscr{O}_{E} \oplus \pi_{2}^{*} \mathscr{O}_{E} \cong \mathscr{O}_{E \times E}^{\oplus 2} .
$$

From the effective divisor $A=\sum n_{i} P_{i}$, let us define the augmented divisor $A^{a}:=\sum\left(n_{i}+1\right) P_{i}$. The differential $d$ induces a map of sheaves on $E \times E$ by just taking derivatives of sections with poles along the divisors $\Delta$ and $E_{P_{i}}$ :

$$
\mathscr{O}_{E \times E}\left(m \Delta+E_{A}\right) \rightarrow \Omega_{E \times E}^{1}\left((m+1) \Delta+E_{A^{a}}\right) \cong\left(\mathscr{O}_{E \times E}\left((m+1) \Delta+E_{A^{a}}\right)\right)^{\oplus 2} .
$$

By projecting to the first summand and applying the functor $\pi_{1 *}$ we get the map of sheaves $\delta: \mathscr{G} \rightarrow$ $\mathscr{U}$, which glues the maps $\delta_{x}$.

The upper bound given by Proposition 4.5 comes from the computation of the Chern classes of the sheaves involved in the picture above. Let us begin this final computation with the following:

Lemma 4.4. For all $m \geq 0$ and for all effective divisors $A$, we have that $c_{1}\left(D_{m, A}\right)=m \cdot \operatorname{deg}(A)$.

Proof. We first check the formula for $n=0$, in which case $A=0$. Consider the short exact sequence

$$
0 \longrightarrow \mathscr{O}_{E \times E}((m-1) \Delta) \longrightarrow \mathscr{O}_{E \times E}(m \Delta) \longrightarrow \mathscr{O}_{\Delta}(\Delta) \longrightarrow 0 .
$$

By the adjunction formula $\mathscr{O}_{\Delta}(\Delta)$ is trivial. If $m \geq 1$, then $R^{1} \pi_{1 *} \mathscr{O}_{E \times E}((m-1) \Delta)=0$ and we get immediately that $c_{1}\left(D_{m, 0}\right)=c_{1}\left(D_{m-1,0}\right)$. For $m=0$ we have

$$
c_{1}\left(D_{0,0}\right)=c_{1}\left(\pi_{1 *} \mathscr{O}_{E \times E}\right)=c_{1}\left(\mathscr{O}_{E}\right)=0 .
$$

Therefore, $c_{1}\left(D_{m, 0}\right)=0$ for all $m \geq 0$.

Assume now $n>0$. After tensoring the short exact sequence (5) with $\mathscr{O}_{E \times E}\left(E_{A}\right)$, we apply the functor $\pi_{1 *}$. Since $n>0$, we have $R^{1} \pi_{1 *} \mathscr{O}_{E \times E}\left((m-1) \Delta+E_{A}\right)=0$ for any $m \geq 1$, therefore the following sequence is exact

$$
0 \longrightarrow D_{m-1, n} \longrightarrow D_{m, n} \longrightarrow \pi_{1 *} \mathscr{O}_{\Delta}\left(E_{A}\right) \longrightarrow 0 .
$$

The first Chern class of $\pi_{1 *} \mathscr{O}_{\Delta}\left(E_{A}\right)$ equals $c_{1}\left(\mathscr{O}_{E}(A)\right)=\operatorname{deg}(A)=n$. Then $c_{1}\left(D_{m, n}\right)=n+c_{1}\left(D_{m-1, n}\right)$ for $n>0$. We can repeat this procedure until we get $c_{1}\left(D_{m, n}\right)=m \cdot n+c_{1}\left(D_{0, n}\right)$.

It remains to take care of the case $m=0$ and $n>0$. Let $P$ be a point in the support of $A$ and set $A_{0}=A \backslash\{P\}$. Similarly to the previous cases, one finds for $n \geq 2$ the following short exact sequence

$$
0 \longrightarrow D_{0, A_{0}} \longrightarrow D_{0, A} \longrightarrow \pi_{1 *} \mathscr{O}_{E_{P}}\left(E_{A}\right) \longrightarrow 0 \text {. }
$$

Since $c_{1}\left(\pi_{1 *} \mathscr{O}_{E_{P}}\left(E_{A}\right)\right)=c_{1}\left(\mathscr{O}_{P}(A)\right)=0$, one gets $c_{1}\left(D_{0, A}\right)=c_{1}\left(D_{0, A_{0}}\right)$. To conclude, we need to show that $c_{1}\left(D_{0, P}\right)=0$ for any point $P \in E$. Indeed we have

$$
0 \longrightarrow D_{0,0} \longrightarrow D_{0, P} \longrightarrow \pi_{1 *} \mathscr{O}_{E_{P}}\left(E_{P}\right) \longrightarrow R^{1} \pi_{1 *} \mathscr{O}_{E \times E} \longrightarrow 0,
$$

which gives that $c_{1}\left(D_{0, P}\right)=c_{1}\left(R^{1} \pi_{1 *} \mathscr{O}_{E \times E}\right)$. By Grothendieck-Verdier duality $R^{1} \pi_{1 *} \mathscr{O}_{E \times E} \cong \mathscr{O}_{E}$ and the result follows.

Proposition 4.5. Equation (4) has at most 16 distinct solutions in degree 4. 
Proof. By Lemma 4.4 we have

$$
\begin{aligned}
& c_{1}(\mathscr{G})=c_{1}\left(D_{3,1}\right)=3, \\
& c_{1}(\mathscr{F})=c_{1}\left(D_{2,1}\right)=2, \\
& c_{1}(\mathscr{U})=c_{1}\left(D_{4,2}\right)=8 .
\end{aligned}
$$

Let us denote the quotient $\mathscr{U} / \mathscr{G}$ by $\mathscr{V}$, we have $c_{1}(\mathscr{V})=c_{1}(\mathscr{U})-c_{1}(\mathscr{G})=5$. By following Grothendieck's notation of $[\mathrm{H}]$, we consider the projective bundle $\mathbb{P}:=\mathbb{P}(\mathscr{F} \vee) \stackrel{q}{\rightarrow} E$ of $\mathscr{F}$. Note that $\operatorname{dim}(\mathbb{P})=3$. Denote the class of the line bundle $\mathscr{O}_{\mathbb{P}}(1)$ by $\epsilon$. The map $f \rightarrow f^{2} \omega d z \bmod \delta(\mathscr{G})$ can be viewed globally as a morphism of vector bundles on $\mathbb{P}$

$$
\phi: \mathscr{O}_{\mathbb{P}}(-2) \rightarrow q^{*} \mathscr{V},
$$

that is, as an element of $H^{0}\left(\mathbb{P}, q^{* \mathscr{V}}(2)\right)$. Recalling that $\epsilon^{3}-q^{*} c_{1}\left(\mathscr{F}^{\vee}\right) \epsilon^{2}=0$, we find $\epsilon^{3}=q^{*} c_{1}\left(\mathscr{F}^{\vee}\right) \epsilon^{2}=$ $-q^{*} c_{1}(\mathscr{F}) \epsilon^{2}=-c_{1}(\mathscr{F})=-2$. If we denote by $A, B$ and $C$ the Chern roots of the rank 3 vector bundles $\mathscr{V}$, we use the splitting principle to compute:

$$
\begin{aligned}
& c_{3}\left(q^{*} \mathscr{V}(2)\right)=(A+2 \epsilon)(B+2 \epsilon)(C+2 \epsilon) \\
& =A B C+2 \epsilon(A B+B C+C A)+4 \epsilon^{2}(A+B+C)+8 \epsilon^{3} \\
& =4 \epsilon^{2} c_{1}(\mathscr{V})+8 \epsilon^{3}=4\left(c_{1}(\mathscr{V})+2 \epsilon^{3}\right)=4(5-2 \times 2)=4 .
\end{aligned}
$$

This happens for each of the 4 spin structures on $E$, thus the equation (4) has at most 16 solutions.

Now we prove the existence of exactly 16 solutions of equation (4) for a particular elliptic curve. The argument is independent of a fixed theta characteristic $\vartheta$ on $E$. Let $g$ be a solution of equation (4). Remember that we defined $\sigma: E \rightarrow E$ to be the involution that fixes the origin $P$ of the elliptic curve. The function $g^{\sigma}:=g \circ \sigma$ is then another odd degree 4 cover triply ramified at $P$. Recall that $Q, R$ and $S$ denote the non-trivial 2-torsion points on $E$.

Lemma 4.6. The solutions $g$ and $g^{\sigma}$ are different.

Proof. Assume $g^{\sigma}= \pm g$. The unique point in $g^{-1}(g(P)) \backslash\{P\}$ is then fixed by $\sigma$ and we may assume this point to be $Q$. Moreover, $\sigma$ acts on the other triple ramification points of $g$, which we denote by $x, y$ and $z$. Since $\sigma$ is an involution, there must be at least one fixed point and we may assume $x=R$. Consider $x^{\prime}$ to be the remaining point in the fibre of $R$, that is $g^{-1}(g(R))=3 R+x^{\prime}$. Then $x^{\prime}$ is also fixed by $\sigma$ and so $x^{\prime}=S$. Summarizing, we have $g^{*}(\infty)=3 P+Q$ and $g^{*}(0)=3 R+S$.

Let $v_{1}, v_{2}$ and $v_{3}$ in $\mathbb{C}$ be the half periods of $E=\mathbb{C} / \Lambda$ and set $e_{i}:=\wp\left(v_{i}\right)$. We consider the equation (2) and (3) from the preliminaries. In particular, $e_{1}+e_{2}+e_{3}=0$. Recall that $\wp$ has a pole of order 2 at 0 and $\wp^{\prime}$ has a pole of order 3 at 0 and on the $v_{i}$. Consider the function $G: \mathbb{C} \rightarrow \mathbb{C}$ defined by

$$
G(z):=\wp^{\prime}(z) \frac{\wp(z)-e_{2}}{\wp(z)-e_{1}} .
$$

The function $G(z)$ has a pole of order 3 at the points 0 and $v_{1}$, and a zero of order 3 at the point $v_{2}$. The half period $v_{1}$ corresponds to the point $Q$ and, similarly, $v_{2}$ corresponds to $R$. We have that $G(-z)=-G(z)$. The attached meromorphic function $g_{0}$ on $E$ satisfies

$$
\operatorname{div}\left(g_{0}\right)=3 P+Q-3 R-S .
$$

Hence up to a constant, $g_{0}=g$. In particular, $G$ has to be an odd function. To impose this we compute the derivative of $G$, then we study the vanishing locus of its discriminant. To simplify calculations, we set

$$
\varphi(z):=\frac{\wp(z)-e_{2}}{\wp(z)-e_{1}},
$$


in such a way that $G(z)=\varphi(z) \wp^{\prime}(z)$. We have

$$
\varphi^{\prime}(z)=\wp^{\prime}(z) \frac{e_{2}-e_{1}}{\left(\wp(z)-e_{1}\right)^{2}} .
$$

We proceed with the computation of $G^{\prime}(z)$ by using the properties of $\wp$ given in equation (2):

$$
\begin{aligned}
G^{\prime}(z) & =\wp^{\prime \prime}(z) \varphi(z)+\wp^{\prime}(z) \varphi^{\prime}(z)=\wp^{\prime \prime}(z) \varphi(z)+\wp^{\prime}(z)^{2} \frac{e_{2}-e_{1}}{\left(\wp(z)-e_{1}\right)^{2}} \\
& =\wp^{\prime \prime}(z) \varphi(z)+4\left(\wp(z)-e_{1}\right)\left(\wp(z)-e_{2}\right)\left(\wp(z)-e_{3}\right) \frac{e_{2}-e_{1}}{\left(\wp(z)-e_{1}\right)^{2}} \\
& =\wp^{\prime \prime}(z) \varphi(z)+\varphi(z)\left(4\left(\wp(z)-e_{3}\right)\left(e_{2}-e_{1}\right)\right)=\varphi(z)\left(\wp^{\prime \prime}(z)+4\left(e_{2}-e_{1}\right)\left(\wp(z)-e_{3}\right)\right) .
\end{aligned}
$$

To understand the ramification of $G$, we study the zeroes of

$$
\wp^{\prime \prime}(z)+4\left(e_{2}-e_{1}\right)\left(\wp(z)-e_{3}\right)=6 \wp(z)^{2}-\frac{1}{2} g_{2}+4\left(e_{2}-e_{1}\right)\left(\wp(z)-e_{3}\right) .
$$

Set $v:=\wp(z)$, and recall that from (3) that

$$
g_{2}=-4\left(e_{1} e_{2}+e_{3}\left(e_{1}+e_{2}\right)\right)=4\left(e_{1}^{2}+e_{1} e_{2}+e_{2}^{2}\right) .
$$

By using (6) and that $e_{3}=-e_{1}-e_{2}$, we finally get the the equation

$$
2\left(3 v^{2}+2\left(e_{2}-e_{1}\right) v+\left(-3 e_{1}^{2}-e_{1} e_{2}+e_{2}^{2}\right)\right)=0,
$$

which has discriminant $\Delta(\tau)=16 \Delta_{0}(\tau)$, where $\Delta_{0}(\tau)=10 e_{1}^{2}-2 e_{2}^{2}+e_{1} e_{2}$. Now we focus on the elliptic curve corresponding to $\tau=i$. For this curve one has $g_{3}=e_{1} e_{2} e_{3}=0$ and $g_{2} \neq 0$. Therefore, one and only one of the values $e_{i}$ is zero. If $e_{1}=0$, then $\Delta_{0}(i)=-2 e_{2}^{2} \neq 0$. If $e_{2}=0$, then $\Delta_{0}(i)=10 e_{1}^{2} \neq 0$. Finally if $e_{3}=0$, then $e_{2}=-e_{1}$ and $\Delta_{0}(i)=8 e_{1}^{2} \neq 0$. We obtain that $g \neq g^{\sigma}$ as desired.

Observe that the elliptic curve $E$ we are considering also has an automorphism $j$ such that $j^{2}=\sigma$. Then $g^{j}$ is a new function with odd ramification. Moreover, $g^{j}=g$ would imply

$$
g^{\sigma}=g^{j \circ j}=g^{j} \circ j=g \circ j=g^{j}=g,
$$

a contradiction. With a similar argument one can prove that $g^{j} \neq g^{\sigma}$ and that all the solutions $g$, $g^{j}, g^{\sigma}, g^{j \sigma}$ are different.

Proposition 4.7. Equation (4) has at least 16 distinct solutions in degree 4.

Proof. For two of the possible theta characteristics, namely the trivial and one of the even ones, we can assume that $j^{*}(\vartheta) \cong \vartheta$. It follows there are exactly 4 solutions in each case: $g, g^{j}, g^{\sigma}=g^{j^{2}}$ and $g^{j \sigma}=g^{j^{3}}$. To show that this is so also for the remaining theta characteristics, we use a monodromy argument. Let us consider the 1-dimensional spin moduli space

$$
\mathscr{S}_{1,1}^{+}=\left\{[E, p, \vartheta]:[E, p] \in \mathscr{M}_{1,1}, \vartheta^{2} \cong \mathscr{O}_{E}, \vartheta ¥ \mathscr{O}_{E}\right\}
$$

which is known to be connected with a forgetful map $\mathscr{S}_{1,1}^{+} \rightarrow \mathscr{M}_{1,1}$ of degree 3 . We have shown that there are 4 odd meromorphic functions corresponding to a general $[E, p, \vartheta] \in \mathscr{S}_{1,1}^{+}$. It follows that for a generic elliptic curve there are 12 solutions attached to even theta characteristics. The conclusion is that we can find at least 16 solutions of Equation (4) for a generic elliptic curve.

4.2. Odd covers of degree 5 on an elliptic curve. We establish the following result:

Theorem 4.8. The number $N_{5}$ of odd maps of degree 5 computed in the case of a general elliptic curve is equal to 16.

Proof. Thanks to Proposition 2.1, this problem is equivalent to finding the number of solutions of equation (4). The result is proved by combining the upper bound provided by Proposition 4.9 and the lower bound provided by Proposition 4.11 . 
This situation is simpler than the one considered in Theorem 4.1, since one of the fibres of the map $f: E \rightarrow \mathbb{P}^{1}$ is $5 P$ and there is no freedom for a new pole. Fix $P$ to be the origin of the curve $E$. We count the solutions of equation (4) for a given spin structure $\vartheta$. Assume $f$ has a pole of order 5 at $P$ and a zero of order 3 at $Q$, as well as two other ramification points of index 3 . By looking at the local expression of $f$ at $P$ and taking derivatives, we obtain that $d f$ has a pole of order 6 at $P$. Derivation induces a map

$$
\delta: H^{0}\left(E, \mathscr{O}_{E}(5 P)\right) \rightarrow H^{0}\left(E, \omega_{E}(6 P)\right) \cong H^{0}\left(E, \mathscr{O}_{E}(6 P)\right) .
$$

Since the kernel is formed by the constants the image of $\delta$ is 4-dimensional. On the one hand, when $\vartheta \cong \mathscr{O}_{E}$, we have to consider the map:

$$
\alpha: H^{0}\left(E, \mathscr{O}_{E}(3 P)\right) \rightarrow H^{0}\left(E, \mathscr{O}_{E}(6 P)\right), \alpha(s):=s^{2} d z .
$$

On the other hand, when $\vartheta$ is even, the map $\alpha$ has to be defined by

$$
\alpha: H^{0}\left(E, \mathscr{O}_{E}(2 P+Q)\right) \rightarrow H^{0}\left(E, \mathscr{O}_{E}(6 P)\right), \quad s \mapsto s^{2} h d z,
$$

where $\operatorname{div}(h)=2 P-2 Q$.

Proposition 4.9. Equation (4) has at most 16 distinct solutions in degree 5.

Proof. The result of Corollary 4.2 still holds: the solutions of Equation (4) lies in the intersection of the images of the maps $\delta$ and $\alpha$, up to constants. Hence, we have to look at the kernel of the map

$$
H^{0}\left(E, \mathscr{O}_{E}(3 P)\right) \rightarrow H^{0}\left(E, \mathscr{O}_{E}(6 P)\right) \rightarrow \frac{H^{0}\left(E, \mathscr{O}_{E}(6 P)\right)}{\delta\left(H^{0}\left(E, \mathscr{O}_{E}(5 P)\right)\right)} .
$$

By projectivizing, this amounts to considering inside $\mathbb{P}\left(H^{0}\left(E, \mathscr{O}_{E}(6 P)\right)\right) \cong \mathbb{P}^{5}$ the intersection of the 3-plane $\mathbb{P}(\operatorname{Im}(\delta))$ with the image $\mathbb{P}\left(H^{0}\left(E, \mathscr{O}_{E}(3 P)\right)\right)$ of $\alpha$, which is a Veronese surface. Counting with multiplicities, there are 4 solutions when $\vartheta$ is trivial. The same argument works also when $\vartheta \neq \mathscr{O}_{E}$. Putting everything together, we obtain $N_{5} \leq 16$.

Now we prove the existence of exactly 16 distinct solutions of equation (4) for a particular elliptic curve. We follow the same strategy as in the previous section. We fix a meromorphic function $g$ inducing an odd map of degree 5 with a pole at $P$ of order 5 and three additional triple ramification points $x, y$ and $z$. Let $\sigma$ be the automorphism of $E$ fixing $P$. Then, $g^{\sigma}$ is another meromorphic function on $E$ with the same properties. Assume $g^{\sigma}= \pm g$. Then one of the ramification points must be one of the points $Q, R, S$. We may assume this point to be $x=Q$ and that $g(Q)=0$.

Lemma 4.10. Let $g$ be a meromorphic function as described above with $g^{\sigma}= \pm g$ and $g(Q)=0$. Then $g^{*}(0)=3 Q+R+S$.

Proof. Assume $u \in E$ is a zero of $g$ which is not fixed by $\sigma$. Then $\sigma(u)$ must be another zero of $g$. Therefore we get $\operatorname{div}(g)=3 Q+u+\sigma(u)-5 P$. Let now consider $\widetilde{G}(z):=\wp^{\prime}(z)\left(\wp(z)-e_{1}\right)$; it is easy to check that the divisor of the meromorphic function $\widetilde{g}$ induced on $E$ by $\widetilde{G}$ is $\operatorname{div}(\widetilde{g})=3 Q+R+S-5 P$. Then, the divisor of $\frac{g}{\widetilde{g}}$ is $u+\sigma(u)-R-S$. Hence $u+\sigma(u) \sim R+S$. Since $u+\sigma(u) \sim 2 R$, we obtain that $R$ and $S$ are linearly equivalent, which is impossible.

From Lemma 4.10 , the functions $g$ and $\widetilde{g}$ have the same attached divisor, hence they only differ by a constant and we may assume that $g=\widetilde{g}$.

Proposition 4.11. Equation (4) has at least 16 solutions in degree 5.

Proof. Now we impose the existence of other odd ramification points for $\widetilde{g}$. We can use the explicit expression of $\widetilde{G}$ and the properties of the derivatives of $\wp$. It is easy to check that

$$
\widetilde{G}^{\prime}(z)=\left(\wp(z)-e_{1}\right)\left(6 \wp(z)^{2}-2\left(e_{1}^{2}+e_{1} e_{2}+e_{2}^{2}\right)+4\left(\wp(z)-e_{2}\right)\left(\wp(z)-e_{3}\right)\right) .
$$


The discriminant of the quadratic part is

$$
\Delta(\tau)=16\left(5 e_{1}^{2}+6 e_{2}^{2}+e_{3}^{2}+5 e_{1} e_{2}-8 e_{2} e_{3}\right) .
$$

As in the computation of $N_{4}$ it turns out that $\Delta(i) \neq 0$ and then $g^{\sigma} \neq g$. Moreover, we can use the involution $j$ with $j^{2}=\sigma$ and then there are 4 different meromorphic functions with the prescribed ramification and the same theta characteristic $\vartheta$ with $j^{*}(\vartheta) \cong \vartheta$. Then we can apply the same monodromy argument we used in the previous section to finish the proof.

\section{COUNTING ODD COVERS OF ELLIPTIC CURVES II: AN APPROACH VIA DEGENERATION}

In this section we present a second proof of Theorems 4.1 and 4.8. The proof relies on counting the number of odd admissible covers from a curve stably equivalent to a rational nodal curve, that is, an elliptic curve with $j$-invariant $\infty$.

5.1. Odd degree 5 covers of elliptic curves. We denote by $\overline{\mathscr{H}}_{1,5}^{\text {ord }}$ the 1 -dimensional Hurwitz space parametrizing odd admissible covers $[f: X \rightarrow \Gamma, P, x, y, z]$, where $X$ (respectively $\Gamma$ ) is a connected nodal curve of arithmetic genus one (respectively zero), $f$ is a finite map of degree 5 which is totally ramified at the point $P \in X$ and triply ramified at the mutually distinct points $x, y, z \in X_{\text {reg }} \backslash\{P\}$. The symmetric group $\mathfrak{S}_{3}$ acts on $\overline{\mathscr{H}}_{1,5}^{\text {ord }}$ by permuting the ramification points $x, y$ and $z$ and we denote the quotient by

$$
\overline{\mathscr{H}}_{1,5}:=\overline{\mathscr{H}}_{1,5}^{\text {ord }} / \mathfrak{S}_{3} \text {. }
$$

Let $\sigma_{5}: \overline{\mathscr{H}}_{1,5} \rightarrow \overline{\mathscr{M}}_{1,1}$ be the map associating to a cover $[f: X \rightarrow \Gamma, P, x+y+z]$ the stabilization of the source curve, that is, [st $(X), P] \in \overline{\mathscr{M}}_{1,1}$. We shall determine the degree of the generically finite morphism $\sigma_{5}$.

We denote by $[R, P, U, V] \in \overline{\mathscr{M}}_{0,3}$ a fixed 3-pointed smooth rational curve and set

$$
\left[E_{\infty}, P\right]:=[R / U \sim V, P] \in \overline{\mathscr{M}}_{1,1}
$$

to be the pointed elliptic curve with $j$-invariant $\infty$. In what follows we explicitly describe the cycle $\sigma_{5}^{*}\left(\left[E_{\infty}, P\right]\right)$. We shall count (with appropriate multiplicities) the admissible covers in $\overline{\mathscr{H}}_{5}$ having as source a nodal curve stably equivalent to $\left[E_{\infty}, P\right]$.

Theorem 5.1. We have that length $\sigma_{5}^{*}\left(\left[E_{\infty}, P\right]\right)=\operatorname{deg}\left(\sigma_{5}\right)=16$. It follows once more that $N_{5}=16$.

Proof. Let $\xi:=[f: X \stackrel{5: 1}{\rightarrow} \Gamma, P, x, y, z] \in \overline{\mathscr{H}}_{1,5}^{\text {ord }}$ be an admissible cover such that the stabilization of $[X, P]$ is $\left[E_{\infty}, P\right]$. In particular $f^{-1}\left(\Gamma_{\text {sing }}\right)=X_{\text {sing }}$, which implies that $R$ appears as an irreducible

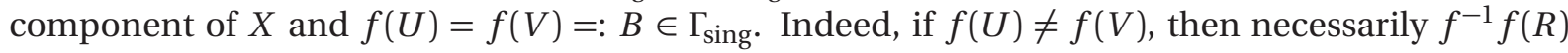
contains another (rational) component of $X$ different from $R$, which is impossible for if we denote $f_{R}:=f_{\mid R}$, then $\operatorname{deg}\left(f_{R}\right)=\operatorname{deg}(f)=5$ because $f_{R}$ is fully ramified at $P$. We denote by $R_{1}$ the subcurve of $X$ meeting $R$ at the points $U$ and $V$. Since the arithmetic genus of $X$ is equal to one, it follows that $R \cap R_{1}=\{U, V\}$. We set $f(R)=: \mathbb{P}^{1}$ and $f\left(R_{1}\right)=: \mathbb{P}_{1}^{1}$, thus $\mathbb{P}^{1} \cap \mathbb{P}_{1}^{1}=\{B\}$.

We claim that the degree of the restriction $f_{1}:=f_{\mid R_{1}}$ is at most 4 and that precisely two of the ramification points $x, y, z$ lie on $R_{1}$, whereas the remaining point lies on $R$. Indeed, else, regarding $B=f(U)=f(V)$ as a smooth point of $\mathbb{P}^{1}=f(R)$, we have $f_{R}^{*}(B)=\alpha \cdot U+(5-\alpha) \cdot V$, where $1 \leq \alpha \leq 4$. Applying the Hurwitz formula to $f_{R}: R \rightarrow \mathbb{P}^{1}$, we obtain that apart from $U, V$ and $P$, the cover $f_{R}$ has precisely one ramification point contributing with multiplicity one to the ramification divisor of $f_{R}$, which is impossible, for $f$ is an odd map. It follows that the fibre $f_{R}^{-1}(B)$ contains a third point $U^{\prime} \in R \backslash\{U, V\}$.

We now turn our attention to the cover $f_{1}: R_{1} \rightarrow \mathbb{P}_{1}^{1}$. We have seen that $\operatorname{deg}\left(f_{1}\right) \leq 4$. If the degree of $f_{1}$ is two or three, then the Hurwitz formula implies that $f_{1}$ has a simple ramification point in 
$R_{1} \backslash\{U, V\}$, which is impossible. Thus $\operatorname{deg}\left(f_{1}\right)=4$ and we write $f_{1}^{*}(B)=\alpha \cdot U+(4-\alpha) \cdot V$, where $1 \leq \alpha \leq 3$. It follows that the map $f_{R}$ is unramified at $U^{\prime}$ and that

$$
f_{R}^{*}(B)=\alpha \cdot U+(4-\alpha) \cdot V+U^{\prime} \in \operatorname{Div}(R) .
$$

Assume $x \in R \backslash\{U, V, P\}$ is the triple ramification point of $f$ lying on $R$, whereas $\{y, z\} \in R_{1} \backslash\{U, V\}$ are the triple ramification point of $f$ lying on $R_{1}$. Note that the curve $X$ consists of a further rational component $R_{2}$ mapping isomorphically onto $f\left(R_{1}\right)$ and meeting $R$ at the point $U^{\prime}$.

We distinguish two cases depending on whether $\alpha \in\{1,3\}$, or $\alpha=2$.

(i) Assume $\alpha=1$, that is, $f_{R}^{*}(B)=U+3 \cdot V+U^{\prime}$ and $f_{1}^{*}(B)=U+3 \cdot V \in \operatorname{Div}\left(R_{1}\right)$. We claim that up to the $P G L(2)$-action on the base, there exists a unique such cover $f_{R}: R \rightarrow \mathbb{P}^{1}$. We may indeed assume $P=\infty \in R, U=1$ and $V=0 \in R$. Then the function

$$
f_{R}(t)=t^{3}(t-1)(t-b),
$$

where $b \in \mathbb{C}$ has a pole of order five at $P$. Imposing the condition that $f$ have triple ramification at a further point $x \in R \backslash\{P, U, V\}$, we obtain that $4 b^{2}-7 b+4=0$, thus there are two choices for $f_{R}$. Observe that these two covers lead to genuinely different points in $\overline{\mathscr{H}}_{1,5}$ (in particular also in $\mathscr{\mathscr { H }}_{1,5}^{\text {ord }}$ ), for $f_{R}$ has no non-trivial automorphisms. Indeed such an automorphism $\tau_{R} \in \operatorname{Aut}(R)$ fixes the point $P$ of total ramification, the unique triple ramification point $x$ of $f_{R}$, as well as the point $U^{\prime} \in f^{-1}(B) \backslash\{U, V\}$. Thus $\tau_{R}=\operatorname{Id}_{R}$.

We now consider the $R_{1}$-side and assume $U=1, V=\infty \in R_{1}$ and $y=0 \in R_{1}$. We may write $f_{1}(t)=\frac{t^{3}(t+a)}{t-1}$. Imposing the condition that $f_{1}$ have a further triple ramification point $z$, we find

$$
f_{1}(t)=\frac{t^{3}(t-4)}{t-1}
$$

and $f_{1}^{*}(0)=3 \cdot 0+1 \cdot 4 \in \operatorname{Div}\left(R_{1}\right)$ and $f_{1}^{*}(-16)=3 \cdot 2+1 \cdot(-2) \in \operatorname{Div}\left(R_{1}\right)$. In particular, $z=2$ is also a triple ramification point of $f_{1}$.

Observe now that $f_{1}$ has an automorphism $\tau_{1}$ of order 2 that fixes the points $U$ and $V$ and interchanges the ramification points $y$ and $z$. Using (7), we find $\tau_{1}(t)=2-t$. This implies that the map $\overline{\mathscr{H}}_{1,5}^{\text {ord }} \rightarrow \overline{\mathscr{H}}_{1,5}$ is ramified with order 2 at such a point $\xi=[f: X \rightarrow \Gamma, P, x, y, z] \in \overline{\mathscr{H}}_{1,5}^{\text {ord }}$.

The following local statement is essential in the proof of both Theorems 5.1 and 5.2 . Claim: The map $\overline{\mathscr{H}}_{1,5}^{\text {ord }} \rightarrow \overline{\mathscr{M}}_{1,1}$ is ramified with order $4=\operatorname{ord}_{U}(f)+\operatorname{ord}_{V}(f)$ at the point $\xi \in \overline{\mathscr{H}}_{1,5}^{\text {ord }}$.

Assuming this fact for a moment, we conclude that the contribution to the cycle $\sigma_{5}^{*}\left(\left[E_{\infty}, P\right]\right)$ coming from the case $\alpha=1$ is equal to $4=2 \times 4 \times \frac{1}{2}=4$ : We multiply by 2 for the two choices of $f_{R}$, by 4 because of the ramification of the map $\overline{\mathscr{H}}_{1,5}^{\text {ord }} \rightarrow \overline{\mathscr{M}}_{1,1}$ at each of the points $\xi$ and divide by 2 because of the existence of the automorphism of $f$ which is trivial along $R$ and $R_{2}$, while being equal to $\tau_{1}$ along $R_{1}$. The case $\alpha=3$ is identical (one switches the role of $u$ and $v$ ). Summarizing the discussion so far, we have identified a subcycle of length $8=4+4$ of $\sigma_{5}^{*}\left(\left[E_{\infty}, P\right]\right)$ coming from the cases $\alpha \in\{1,3\}$.

Proof of the claim. We show that $\overline{\mathscr{H}}_{1,5}^{\text {ord }} \rightarrow \overline{\mathscr{M}}_{1,1}$ is ramified with order 4 at the point $\xi$. Let

$$
F: \mathscr{X} \rightarrow \mathscr{P}
$$

be the universal degree 5 admissible cover over $\overline{\mathscr{H}}_{1,5}^{\text {ord }}$. One has a finite map $\mathfrak{b}: \overline{\mathscr{H}}_{1,5}^{\text {ord }} \rightarrow \overline{\mathscr{M}}_{0,4}$ associating to an admissible cover $[f: X \rightarrow \Gamma, P, x, y, z]$ the point $[\Gamma, f(P), f(x), f(y), f(z)] \in \overline{\mathscr{M}}_{0,4}$. According to the local description (1) of the local ring of $\overline{\mathscr{H}}_{1,5}^{\text {ord }}$ at the point $\xi$, we have that

$$
\hat{\mathscr{O}}_{\xi, \overline{\mathscr{H}}_{1,5}^{\mathrm{ord}}} \cong \mathbb{C}\left[\left[s_{1}, s_{2}\right]\right] / s_{1}^{3}=s_{2}=t,
$$


where $t$ is the local parameter on $\overline{\mathscr{M}}_{0,4}$ corresponding to the boundary point $\mathfrak{b}(\xi)$. Around the points $(\xi, V)$ and $(\xi, U) \in \mathscr{X}$, the cover $F$ considered in (8) has the following local expression:

$\mathscr{X}$ around $(\xi, V): v_{1} v_{2}=s_{1}, \mathscr{P}$ around $F(\xi, V): \gamma_{1} \gamma_{2}=s_{1}^{3}$, the map $F: \gamma_{1}=v_{1}^{3}, \gamma_{2}=v_{2}^{3}$, respectively

$\mathscr{X}$ around $(\xi, U): u_{1} u_{2}=s_{1}, \quad \mathscr{P}$ around $F(\xi, U): \gamma_{1} \gamma_{2}=s_{1}$, the map $F: \gamma_{1}=u_{1}, \gamma_{2}=u_{2}$.

We consider the map Spec $\mathbb{C}[[t]] \rightarrow \hat{\mathscr{O}}_{\xi, \overline{\mathscr{H}}_{1,5}^{\text {ord }}}$ given by sending $t \mapsto\left(s_{1}=t, s_{2}=t^{3}\right)$. The induced family of curves $\mathscr{X} \times \overline{\mathscr{H}}_{1,5}^{\text {ord }}$ Spec $\mathbb{C}[[t]] \rightarrow \operatorname{Spec} \mathbb{C}[[t]]$ has local equation $v_{1} v_{2}=t$ around the point $(\xi, V)$, and $u_{1} u_{2}=t^{3}$ around the point $(\xi, U)$ respectively. The fibre over 0 consists of the nodal genus one curve $X=R \cup R_{1} \cup R_{2}$. We first blow-down the (-1)-curve $R_{2}$ and then $R_{1}$. The resulting curve $\mathscr{X}^{\prime} \rightarrow$ Spec $\mathbb{C}[[t]]$ is the family of curves induced by base-change from $F$ under the map $\overline{\mathscr{H}}_{1,5}^{\text {ord }} \rightarrow \overline{\mathscr{M}}_{1,1}$. Its central fibre is $\left[E_{\infty}, P\right]$ and the local equation of $\mathscr{X}^{\prime}$ around the unique node of the central fibre is

$$
v_{1} v_{2}=t \cdot t^{3}=t^{4}
$$

which finishes the proof of the claim.

We now proceed with the proof of the remaining cases of Theorem 5.1,

(ii) Assume now $\alpha=2$, thus $f_{R}^{*}(B)=2 \cdot U+2 \cdot V+U^{\prime} \in \operatorname{Div}(R)$ and $f_{1}^{*}(B)=2 \cdot U+2 \cdot V \in \operatorname{Div}\left(R_{1}\right)$. In order to count the number of such maps $f_{R}$, assume again $P=\infty \in R, U=1$ and $V=0 \in R$. Writing $f_{R}(t)=t^{2}(t-1)^{2}(t-b)$, the condition that $f_{R}$ has a triple ramification point $x \in R \backslash\{0,1, \infty\}$ leads to the equation $16 b^{2}-16 b+9=0$, thus to two choices for $f_{R}$. The same argument as in the case (i) shows that $f_{R}$ has no automorphism, nor are the found maps equivalent under the $P G L(2)$-action. We now consider the $R_{1}$-side and set $U=1, V=0 \in R_{1}$ and $y=\infty \in R_{1}$. Up to the $P G L(2)$-action on the base $\mathbb{P}_{1}^{1}$ of the map $f_{1}: R_{1} \rightarrow \mathbb{P}_{1}^{1}$ we find two solutions, namely

$$
f_{1}(t)=\frac{48 \sqrt{3} t^{2}(t-1)^{2}}{(-2 t+1+\sqrt{3})(\sqrt{3}+6 t-3)^{3}} \text { and } \tilde{f}_{1}(t)=\frac{t^{2}(t-1)^{2}}{t-\frac{1}{2}-\frac{\sqrt{3}}{4}} \text {. }
$$

Neither $f_{1}$ nor $\tilde{f}_{1}$ have non-trivial automorphisms. Denote by $\tau_{1}: R_{1} \rightarrow R_{1}$ the automorphism fixing 0 and 1 and such that

$$
\tau_{1}(\infty)=\frac{1}{2}+\frac{\sqrt{3}}{6} \text { and } \tau_{1}\left(\frac{1}{2}-\frac{\sqrt{3}}{6}\right)=\infty
$$

Then $\tilde{f}_{1} \circ \tau_{1}=f_{1}$. Via the automorphism $\tau \in \operatorname{Aut}(X)$ such that $\tau_{R}=\operatorname{Id}_{R}$ and $\tau_{\mid R_{1}}=\tau_{1}$, it follows that $f_{1}$ and $\tilde{f}_{1}$ lead to the same point of $\overline{\mathscr{H}}_{1,5}^{\text {ord }}$. An argument identical to the one in the claim shows that around each such point $\xi$, the map $\overline{\mathscr{H}}_{1,5}^{\text {ord }} \rightarrow \overline{\mathscr{M}}_{1,1}$ is ramified with order 4 . Summarizing, the contribution to the cycle $\sigma_{5}^{*}\left(\left[E_{\infty}, P\right]\right)$ coming from case (ii) is equal to $2 \times 4=8$.

None of the points $\xi \in \overline{\mathscr{H}}_{1,5}^{\text {ord }}$ found in this proof carry an automorphism fixing all the branch points, hence they all correspond to smooth points of $\overline{\mathscr{H}}_{1,5}^{\text {ord }}$. Putting cases (i) and (ii) together, we conclude that the degree of the map $\sigma_{5}$ equals $16=8+8$, which finishes the proof.

5.2. Odd degree 4 covers of elliptic curves. We denote by $\overline{\mathscr{H}}_{1,4}^{\text {ord }}$ the 1 -dimensional Hurwitz space parametrizing odd admissible covers $[f: X \rightarrow \Gamma, P, x, y, z]$, where $X$ (respectively $\Gamma$ ) is a connected nodal curve of arithmetic genus one (respectively zero), $f$ is a finite map of degree 4 which is triply 
ramified at the point $P \in X$ and at the pairwise distinct points $x, y, z \in X_{\mathrm{reg}} \backslash\{P\}$. The symmetric group $\mathfrak{S}_{3}$ acts on $\overline{\mathscr{H}}_{1,4}^{\text {ord }}$ by permuting $x, y$ and $z$. Let

$$
\overline{\mathscr{H}}_{1,4}:=\overline{\mathscr{H}}_{1,4}^{\text {ord }} / \mathfrak{S}_{3}
$$

be the quotient and let $\sigma_{4}: \overline{\mathscr{H}}_{1,4} \rightarrow \overline{\mathscr{M}}_{1,1}$ be the map associating to a cover $[f: X \rightarrow \Gamma, P, x+y+z]$ the stabilization of the source curve.

Theorem 5.2. We have that length $\sigma_{4}^{*}\left(\left[E_{\infty}, P\right]\right)=\operatorname{deg}\left(\sigma_{4}\right)=16$. It follows once more that $N_{4}=16$.

Proof. We proceed along the lines of the proof of Theorem 5.1, highlighting the things that are different. We start with an admissible cover $\xi:=[f: X \stackrel{4: 1}{\rightarrow} \Gamma, P, x, y, z] \in \overline{\mathscr{H}}_{1,4}^{\text {ord }}$ such that the stabilization of $[X, P]$ is $\left[E_{\infty}, P\right]$. As before, $R$ appears as an irreducible component of $X$ and $f(U)=f(V)=$ : $B \in \Gamma_{\text {sing }}$. We denote by $R_{1}$ the subcurve of $X$ meeting $R$ precisely at the points $U$ and $V$. We set $f_{R}:=f_{\mid R}: R \rightarrow \mathbb{P}^{1}$ and $f_{1}:=f_{\mid R_{1}}: R_{1} \rightarrow \mathbb{P}_{1}^{1}$, where $\Gamma=\mathbb{P}^{1} \cup_{B} \mathbb{P}_{1}^{1}$.

There are three types of admissible covers possible for $\xi$. First, we could have $\operatorname{deg}\left(f_{R}\right)=\operatorname{deg}\left(f_{1}\right)=4$ and $f_{R}^{*}(B)=\alpha \cdot U+(4-\alpha) \cdot V \in \operatorname{Div}(R)$ and $f_{1}^{*}(B)=\alpha \cdot U+(4-\alpha) \cdot V \in \operatorname{Div}\left(R_{1}\right)$. Let $x \in R \backslash\{P, U, V\}$ be the remaining triple ramification point of $f_{R}$ and we denote by $\{y, z\} \subseteq R_{1} \backslash\{U, V\}$ the remaining triple ramification points of $f_{1}$.

(i) $\alpha=1$. Setting $P=0, U=1, V=\infty \in R$, we find a unique solution for $f_{R}$, the one given by (7)

$$
f_{R}(t)=\frac{t^{3}(t-4)}{t-1}
$$

The cover $f_{R}$ has no automorphism, for such an automorphism $\tau_{R}$ would have to fix both the marked point $P$, as well as $U$ an $V$, hence $\tau_{R}=\operatorname{Id}_{R}$. On the $R_{1}$-side, setting $U=1, V=\infty \in R_{1}$ and $y=0$, we have a unique choice for $f_{1}$ given by the same formula (7). However, in this case, as in the proof of Theorem 5.1, $f_{1}$ does have an automorphism $\tau_{1} \in \operatorname{Aut}\left(R_{1}\right)$ which fixes both points $U$ and $V$ and interchanges the ramification points $y$ and $z$. The map $\overline{\mathscr{H}}_{1,4}^{\text {ord }} \rightarrow \overline{\mathscr{M}}_{1,1}$ is ramified with order 4 at the point $\xi$. All in all, one gets a contribution of $4=2 \times 4 \times \frac{1}{2}$ to the cycle $\sigma_{4}^{*}\left(\left[E_{\infty}, P\right]\right)$ coming from the case when $\alpha \in\{1,3\}$. The factor $\frac{1}{2}$ is explained by the simple ramification of the map $\overline{\mathscr{H}}_{1,4}^{\text {ord }} \rightarrow \overline{\mathscr{H}}_{1,4}$ at the point $\xi$.

(ii) $\alpha=2$. Setting $U=0, V=1 \in R_{1}$ and $y=\infty \in R_{1}$, following (9) we find two solutions for $f_{1}$, which are related via the $P G L(2)$-action on $R_{1}$. On the $R$-side, we set also $U=0, V=1 \in R$ and $P=\infty \in R$, by using once more (9) we find two solutions for $f_{R}$, which this time are not equivalent to one another, for an automorphism $\tau_{R}$ has to fix $U, V$, as well as $P$, hence $\tau_{R}=\operatorname{Id}_{R}$. All in all, we get a contribution of $2 \times 4=8$ to the cycle $\sigma_{4}^{*}\left(\left[E_{\infty}, P\right]\right)$, where the factor 4 equals the ramification index of the map $\overline{\mathscr{H}}_{1,4}^{\text {ord }} \rightarrow \overline{\mathscr{M}}_{1,1}$ at each of the points $\xi$ considered.

(iii) This is the situation which has no equivalent in the proof of Theorem 5.1. In this case $\operatorname{deg}\left(f_{R}\right)=3$ and $\operatorname{deg}\left(f_{1}\right)=3$. The components $R$ and $R_{1}$ meet at the points $U$ and $V$ and $f^{-1}(B)=\left\{U, V, U^{\prime}, V^{\prime}\right\}$, where $\left\{U^{\prime}\right\}=R \cap R_{3}$ and $\left\{V^{\prime}\right\}=R_{1} \cap R_{2}$. Here $R_{2}$ and $R_{3}$ are smooth rational curves mapping isomorphically onto $\mathbb{P}^{1}=f(R)$ and $\mathbb{P}_{1}^{1}=f\left(R_{1}\right)$ respectively. Thus $f^{-1}\left(\mathbb{P}^{1}\right)$ is the disjoint union of $R$ and $R_{2}$, whereas $f^{-1}\left(\mathbb{P}_{1}^{1}\right)$ is the disjoint union of $R_{1}$ and $R_{3}$. Note that $f$ is unramified over the node $B$ of $\Gamma$.

Modulo the $P G L(2)$-action, there are two choices for a map $f_{R}: R \rightarrow \mathbb{P}^{1}$ of degree 3 triply ramified at $P$ and at a further unspecified point $x \in R \backslash\{P\}$ and satisfying $f_{R}(U)=f_{R}(V)$. In coordinates, if we set $P=\infty, U=1$ and $V=0$, then the two choices are

$$
f_{R}(t)=\left(t-\frac{1}{2}+i \frac{\sqrt{3}}{6}\right)^{3} \text { and } \tilde{f}_{R}(t)=-\left(t-\frac{1}{2}-i \frac{\sqrt{3}}{6}\right)^{3} .
$$


Observe that if $\tau_{R} \in \operatorname{Aut}(R)$ is the automorphism given by $\tau_{R}(t)=1-t$, thus $\tau_{R}(U)=V, \tau_{R}(V)=U$ and $\tau_{R}(P)=P$, then $\tilde{f}_{R}=f_{R} \circ \tau_{R}$. The same applies for the component $R_{1}$ of $X$. There are two ways, say $f_{1}$ and $\tilde{f}_{1}$ of choosing a degree 3 map triply ramified at both $y$ and $z$ and having $U$ and $V$ in the same fibre. The maps $f_{1}$ and $\tilde{f}_{1}$ are related by an automorphism $\tau_{1}$ of $R_{1}$ which interchanges $U$ and $V$ and fixes $y$. We find that in total there are there are $t w o$ points in $\overline{\mathscr{H}}_{1,4}$ of this type in $\sigma_{4}^{-1}\left(\left[E_{\infty}, P\right]\right)$.

A similar calculation like in the Claim in the proof of Theorem 5.1 shows that the map $\overline{\mathscr{H}}_{1,4}^{\text {ord }} \rightarrow$ $\overline{\mathscr{M}}_{1,1}$ is ramified to order 2 at both these points. All in all, we have a contribution of $4=2 \times 2$ to the cycle $\sigma_{4}^{-1}\left(\left[E_{\infty}, P\right]\right)$ coming from case (iii).

Summarizing cases (i), (ii), (iii), we find that length $\sigma_{4}^{*}\left(\left[E_{\infty}, P\right]\right)=4+8+4=16$, which finishes the proof.

\section{THE GENERATING SERIES OF ALTERNATING CATALAN NUMBERS}

In this Section we explain how using basic facts from Schubert calculus coupled with the Lagrange Inversion formula one can derive from Theorem 3.2 both Theorems 1.1]and 1.2.

We fix $V:=\mathbb{C}^{2 g+2}$ and set $\mathbb{G}:=G(2, V)$. Recall the notation $\sigma_{\alpha_{1}, \alpha_{0}}$ for the Schubert cycle in $\mathbb{G}$. We write $\sigma_{\alpha}:=\sigma_{\alpha a, 0}$ for each $\alpha \geq 1$. It is well-known that $\sigma_{1}$ is a hyperplane section of $\mathbb{G}$ in its Plücker embedding. In particular, $C_{2 g}=\sigma_{1}^{2 g}=\operatorname{deg}(\mathbb{G})=\frac{1}{2 g+1}\left(\begin{array}{l}4 g \\ 2 g\end{array}\right)$. We also recall Giambelli's formula $\sigma_{\alpha_{1}, \alpha_{0}}=\sigma_{\alpha_{1}} \cdot \sigma_{\alpha_{0}}-\sigma_{\alpha_{1}+1} \cdot \sigma_{\alpha_{0}-1} \in H^{*}(\mathbb{G}, \mathbb{Z})$.

It is also known that $H^{*}(\mathbb{G}, \mathbb{Z})$ is generated by the classes $\sigma_{1}$ and $\sigma_{2}$ and the top intersection products involving these two classes are given by the following formula, see e.g. [O] Remark 3.4

$$
\sigma_{1}^{2 m} \sigma_{2}^{2 g-2 m}=\sum_{i=0}^{2 g-m}(-1)^{i}\left(\begin{array}{c}
2 g-m \\
i
\end{array}\right) C_{2 g-i} .
$$

We are now in a position to prove Theorem 1.1

Proof of Theorem 1.1. Since we have shown that $N_{4}=N_{5}=16$, Theorem 3.2 can be rewritten as

$$
\mathfrak{A}_{g}=16^{g}\left(\sigma_{4}+\sigma_{3,1}\right)^{g} \text {. }
$$

We are going to rewrite this expression in terms involving only the products appearing in (10). Firstly, Giambelli's formula yields $\sigma_{4}+\sigma_{3,1}=\sigma_{1} \sigma_{3}$. Applying Giambelli's formula once more, we obtain $\sigma_{3}=\sigma_{1} \sigma_{2}-\sigma_{2,1}=\sigma_{1}\left(\sigma_{2}-\sigma_{1,1}\right.$, where for the last equality we have used Pieri's formula. One final application of Giambelli's formula yields $\sigma_{1,1}=\sigma_{1}^{2}-\sigma_{2}$, implying $\sigma_{3}=2 \sigma_{1} \sigma_{2}-\sigma_{1}^{3}$. Thus

$$
\begin{aligned}
& \sigma_{1}^{g} \sigma_{3}^{g}=\sigma_{1}^{2 g}\left(2 \sigma_{2}-\sigma_{1}^{2}\right)^{g}=\sum_{k=0}^{g} \sum_{i=0}^{g-k}(-1)^{k+i} 2^{g-k}\left(\begin{array}{l}
g \\
k
\end{array}\right)\left(\begin{array}{c}
g-k \\
i
\end{array}\right) C_{2 g-i} \\
= & \sum_{i=0}^{g}(-1)^{i}\left(\sum_{k=0}^{g-k}(-1)^{k} 2^{g-k}\left(\begin{array}{c}
g \\
k
\end{array}\right)\left(\begin{array}{c}
g-k \\
i
\end{array}\right)\right) C_{2 g-i}=\sum_{i=0}^{g}(-1)^{i} 2^{i}\left(\begin{array}{l}
g \\
i
\end{array}\right) C_{2 g-i},
\end{aligned}
$$

where we have used the identity $\sum_{k=0}^{g-i}(-1)^{k} 2^{g-k}\left(\begin{array}{c}g \\ k\end{array}\right)\left(\begin{array}{c}g-k \\ i\end{array}\right)=\left(\begin{array}{c}g \\ i\end{array}\right) 2^{i}$. This brings the proof to an end.

6.1. Lagrange inversion for alternating Catalan numbers. In order to determine the generating function of the alternating Catalan numbers we use Lagrange inversion. The help of D. Oprea in this section is gratefully acknowledged.

For a power series $f(w)=\sum_{n \geq 0} a_{n} w^{n} \in \mathbb{Q}[[w]]$, we denote its coefficients by $\left[w^{n}\right] f(w):=a_{n}$. Suppose one can find two power series $\psi(z)$ and $\phi(z)$ with $\left[z^{0}\right] \phi(z) \neq 0$, such that the function 
$f(w)=\sum_{n \geq 0} a_{n} w^{n}$ can be written as

$$
f(w)=\sum_{n \geq 0} w^{n}\left(\left[z^{n}\right]\left(\psi(z) \phi^{n}(z)\right)\right) .
$$

Then there exists a unique power series $u=u(w)$ such that $u(w)=w \phi(u(w))$. Moreover one has

$$
f(w)=\frac{\psi(u)}{\phi(u)} \cdot \frac{d u}{d w}=\frac{\psi(u)}{1-w \phi^{\prime}(u)},
$$

where we refer to [G] 1.2.4 for further details and examples.

We shall now bring the generating function of the alternating Catalan numbers to the form (11). To that end, for any $a \in \mathbb{R}$ we introduce the symbol

$$
\left(\begin{array}{l}
a \\
n
\end{array}\right):=\frac{a(a-1) \cdots(a-n+1)}{n !}
$$

thus we have $(1+z)^{a}=\sum_{n \geq 0}\left(\begin{array}{l}a \\ n\end{array}\right) z^{a}$. With this notation, we observe that the Catalan numbers $C_{n}$ can be rewritten as

$$
C_{n}=\frac{1}{n+1}\left(\begin{array}{c}
2 n \\
n
\end{array}\right)=(-1)^{n} 2^{2 n+1}\left(\begin{array}{c}
\frac{1}{2} \\
n+1
\end{array}\right) .
$$

Using the expression of the alternating Catalan numbers from Theorem 1.1 we then have

$$
\begin{gathered}
\mathfrak{A}_{g}=16^{g} \sum_{s=0}^{g}(-2)^{s}\left(\begin{array}{l}
g \\
s
\end{array}\right) C_{2 g-s}=\sum_{s=0}^{g} 2^{8 g-s+1}\left(\begin{array}{l}
g \\
s
\end{array}\right)\left(\begin{array}{c}
\frac{1}{2} \\
2 g-s+1
\end{array}\right) \\
=\left[z^{2 g+1}\right] 2^{8 g+1}\left(1+\frac{z}{2}\right)^{g}(1+z)^{\frac{1}{2}} .
\end{gathered}
$$

We can now complete the proof of Theorem 1.2 . Proof of Theorem 1.2 . We introduce the auxiliary functions

$$
\phi(z)=16\left(1+\frac{z}{2}\right)^{\frac{1}{2}} \text { and } \psi(z)=\frac{1}{8}(1+z)^{\frac{1}{2}}\left(1+\frac{z}{2}\right)^{-\frac{1}{2}},
$$

then form the function $f(w)=\sum_{n \geq 0} w^{n}\left[z^{n}\right]\left(\phi^{n}(z) \psi(z)\right)$. Then the function $h(w):=\frac{1}{2}(f(w)-$ $f(-w))$ retaining only the odd coefficients of $f$ can be rewritten as

$$
h(w)=\sum_{g \geq 0} w^{2 g+1}\left[z^{2 g+1}\right]\left(1+\frac{z}{2}\right)^{g}\left(1+\frac{z}{2}\right)^{-\frac{1}{2}} \cdot 2^{4(2 g+1)-3}=\sum_{g \geq 0} \mathfrak{A}_{g} w^{2 g+1},
$$

that is, $h(w)$ is the generating function of all alternating Catalan numbers. In order to apply (12), we introduce the function $u=u(w)$ such that $u=w \phi(u)$, from which we find

$$
w=\frac{u}{16 \sqrt{1+\frac{u}{2}}}, \quad \text { or equivalently } \quad u=16\left(4 w+\sqrt{16 w^{2}+1}\right) .
$$

We compute $\frac{d w}{d u}=\frac{u+4}{32(u+2) \sqrt{1+\frac{u}{2}}}$, hence the Lagrange inversion formula (12) leads to

$$
f(w)=\frac{\sqrt{(1+u)\left(1+\frac{u}{2}\right)}}{2(u+4)},
$$

or equivalently

$$
f(w)=\frac{\sqrt{64 w^{2}+1+16 w \sqrt{16 w^{2}+1}}}{8 \sqrt{16 w^{2}+1}}
$$

which leads to the claimed formula for $h(w)=\frac{1}{2}(f(w)-f(-w))$. 


\section{REFERENCES}

[ACV] D. Abramovich, A. Corti And A. Vistoli, Twisted bundles and admissible covers, Comm. Algebra 31 (2003), 3547-3618.

[AMS] M. ABRAMOWITZ, I.A. STEGUN, EDS., Handbook of mathematical functions, with formulas, graphs and mathematical tables, Applied Mathematics Series 55 (1966).

[AP] M. ARtebani AND G.P. Pirola, Algebraic functions with even monodromy, Proc. Amer.Math.Soc. 133 (2005), 331341.

[C] G. Castelnuovo, Numero delle involuzioni razionali giacenti sopra una curva di dato genere, Rendiconti R. Accad. Lincei, 5 (1889), 130-133.

[Di] S. DIAZ, Exceptional Weierstrass points and the divisor on moduli space that they define, Memoirs of the American Math. Soc. 327 (1985).

[EH1] D. EISENBUD AND J. HARRIS, Divisors on general curves and cuspidal rational curves, Invent. Math. 74 (1983), 371418.

[EH2] D. EisenbUd AND J. HARRIS, Limit linear series: basic theory, Invent. Math. 85 (1986), 337-371.

[FS] P. Flajolet AND R. SEDGewick, Analytic combinatorics, Cambridge University Press 2009.

[F1] M.D. FRIED, Alternating groups and moduli space lifting invariants, Israel J. Math. 179 (2010), 57-125.

[GM] R. GURALNICK AND K. MAGAARD, On the minimal degree of a primitive permutation group, J. Algebra 207 (1998), $127-145$.

[GS] R. GURALNICK AND J. SHARESHIAN, Symmetric and alternating groups as monodromy groups of Riemann surfaces, Memoirs of the American Math. Soc. 189 (2007).

[GN] R. GuRAlnick AND M. NeUBAuER, Monodromy groups of branched coverings: the generic case, Contemporary Mathematics 186 (1995), 325-352.

[GJ] I. Goulden And D. JACKson, Combinatorial enumeration, Dover Books of Mathematics 2004.

[HM] J. HARRIS AND D. MuMFORD, On the Kodaira dimension of the moduli space of curves, Inventiones Math 67 (1982), 23-86.

[H] R. HARTSHorne, Algebraic Geometry, Springer-Verlag (1977).

[La] S. LANG, Elliptic functions, Addison-Wisley Publishing Company Inc (1973).

[Li] C. LIAN, Enumerating pencils with moving ramification on curves, arXiv:1907.09087

[MV] K. MAGAARD AND H. VÖLKLEIN, The monodromy group of a function on a general curve, Israel J. Math. 141 (2004), 355-368.

[MTV] E. MukHin, V. TARASOv AND A. VARChEnKo, Schubert calculus and representations of the general linear group, Journal of the American Math. Soc. 22 (2009), 909-940.

[Mu] D. Mumford, Theta-characteristics on an algebraic curve, Ann. Sci. École Normale Sup. 4 (1971), 181-192.

[O] T.C. Oliveira, Catalan traffic and integrals on the Grassmannian of lines, Discrete Math. 308 (2008), 148-152.

[S1] J.P. SERrE, Relèvements dans $\tilde{A}_{n}$, C. R. Acad. Sci. Paris Sér. I Math. 311 (1990), 477-482.

[S2] J.P. SERRE, Revêtements à ramification impaire et thêta-caractéristiques. C. R. Acad. Sci. Paris Sér. I Math. 311 (1990), 547-552.

[St] R. Stanley, Enumerative combinatorics, Volume II, Cambridge Studies in Advanced Mathematics 62.

GAVRIL FARKAS: InSTITUT FÜR MATHEMATIK, Humboldt UNIVERSITÄT ZU BERLIN

UNTER DEN LINDEN 6, 10099 BERLIN, GERMANY

E-mail address: farkas@math.hu-berlin.de

Riccardo Moschetti: Dipartimento di Matematica, Universit‘a degli Studi di Pavia

Via FERRATA, 1, 27100 PAVIA, ITALY

E-mail address: rmoschetti@gmail.com

Juan Carlos Naranjo: Universitat de Barcelona, Departament de Matem'atiquesi Inform'atica

GRAN VIA 585, 08007 BARCELONA, SPAIN

E-mail address: jcnaranjo@ub.edu

Gian Pietro Pirola: Dipartimento di Matematica, Universit'a Degli Studi di Pavia

Via FERRATA, 1, 27100 PAVIA, ITALY

E-mail address: gianpietro.pirola@unipv . it 